\title{
ON THREE DIMENSIONAL MULTIVARIATE VERSION OF Q-NORMAL DISTRIBUTION AND PROBABILISTIC INTERPRETATIONS OF ASKEY-WILSON, AL-SALAM-CHIHARA AND Q-ULTRASPHERICAL POLYNOMIALS .
}

\author{
PAWEŁ J. SZABŁOWSKI
}

\begin{abstract}
We study properties of compactly supported, 4 parameter $\left(\rho_{12}, \rho_{23}, \rho_{13}, q\right) \in(-1,1)^{\times 4}$ family of continuous type 3 dimensional distributions, that have the property that for $q \rightarrow 1^{-}$this family tends to some 3 dimensional Normal distribution. For $q=0$ we deal with 3 dimensional generalization of Kesten-McKay distribution. In a very special case when $\rho_{12} \rho_{13} \rho_{23}=q$ all one dimensional marginals are identical, semicircle distributions. We find both all marginal as well as all conditional distributions. Moreover, we find also families of polynomials that are orthogonalized by these one-dimensional margins and one-dimensional conditional distributions. Consequently, we find moments of both conditional and unconditional distributions of dimensions one and two. In particular, we show that all one-dimensional and two-dimensional conditional moments of, say, order $n$ and are polynomials of the same order $n$ in the conditioning random variables. Finding above mentioned orthogonal polynomials leads us to a probabilistic interpretation of these polynomials. Among them are the famous Askey-Wilson, Al-SalamChihara polynomials considered in the complex, but conjugate, parameters, as well as q-Hermite and Rogers polynomials. It seems that this paper is one of the first papers that give a probabilistic interpretation of Rogers (continuous q-ultraspherical) polynomials.
\end{abstract}

\section{INTRODUCTION}

The purpose of this note is double. Firstly, to present a further step in the search for the non trivial generalizations of the Normal (Gaussian) density. The unique properties of the Normal distribution are well known and found to be useful in many, not only stochastic, applications. Let us mention only one such property that is in fact well known, however, is less popularized and which is one of the most useful. Namely, all conditional mixed moments are polynomials of the same order in the conditioning random variables. To formulate it precisely, let us introduce the following notation. Let $\mathcal{F}$ denote some set of indices, then $\sigma\left(X_{j}, j \notin \mathcal{F}\right)$ we mean the $\sigma$-field generated by all random variables $X_{j}$ whose indices do not belong the set $\mathcal{F}$. Now the property in question can be expressed in the precise fashion. Let us assume that a vector $\left(X_{1}, \ldots, X_{n}\right)$ has joint Normal distribution. Let $\mathcal{F}$ be a subset of $\{1, \ldots, n\}$, then the conditional expectation given by the expression

Date: September 2017 .

2010 Mathematics Subject Classification. Primary 62H86, 60E99; Secondary 05A30, 33D45.

Key words and phrases. 3 dimensional density, Conditional distribution, Moments, Conditional Moments, Orthogonal polynomials. Al-Salam-Chihara, Rogers, Askey-Wilson polynomials. 
$E\left(\prod_{i \in \mathcal{F}} X_{i}^{m_{i}} \mid \sigma\left(X_{j}, j \notin \mathcal{F}\right)\right)$ is a polynomial of order $\sum_{i \in \mathcal{F}} m_{i}$ in $X_{j}, j \notin \mathcal{F}$. The above mentioned property happens to all nontrivial subsets $\mathcal{F}$ that is why we will say that Normal distribution has polynomial conditional moments property (PCM).

This beautiful property has numerous applications is the theory of Gaussian stochastic processes. In particular, is strongly reflected in the properties of Wiener and related processes whose role in the contemporary analysis is difficult to overestimate.

The second intention of this note is to give a probabilistic interpretation of certain, well known in other areas of mathematical analysis, families of orthogonal polynomials. More precisely, we mean certain families of polynomials that appear in the so called $q$-series theory and are related to the so called Askey-Wilson polynomials. There were many interpretations of these polynomials in combinatorics (to mention only works of Sylvie Corteel). It was a great surprise to R. Askey to discover that the so called $q$-Hermite polynomials have nice probabilistic interpretation shown in the work of W. Bryc [2]. To the astonishment of R. Askey there was no $q$ in the original formulation of the probabilistic problem. Later using the same model Bryc, Matysiak and Szabłowski were able to interpret probabilistically the so called Al-Salam-Chihara polynomials. For details, see [9].

We are not that smart as W. Bryc and are not able to eliminate a variable $q$ from the formulation of the problem. However, we are able to define a threedimensional, compactly supported, continuous type distribution, calculate its all marginal unconditional as well as conditional distributions. Moreover, we are able to find conditional moments and show that the analyzed distribution has PCM property.

We are also able to find polynomials that are orthogonalized by the one-dimensional marginals (Rogers polynomials) and by the one-dimensional conditional $X \mid(Y=$ $y, Z=z$ ) (Askey-Wilson polynomials).

We notice also that as the two-dimensional marginals appear products of the so called $q$-conditional normal $(q-\mathrm{CN})$ distribution analyzed say in 4 and, as mentioned above, as one-dimensional marginal Rogers distribution. As a special case we get either the so called generalized Kesten-McKay (recently analyzed in 12]) or the semicircle distributions. Both of them have numerous applications. Especially the semicircle distribution and related distributions lie in the center of the so called free probability a branch of non-commutative probability recently dynamically developing.

The paper is organized as follows. In the next section we introduce the analyzed density. Next we introduce notation that describes it briefly. Then in subsection 2.2 we provide definitions of the families of polynomials that will appear in the sequel and also some auxiliary facts concerning them.

Section, 3 presents our main results. It consists of two subsections the first one presenting marginal distributions and the families of polynomials that are orthogonal with respect to them and the second presenting conditional distributions and families of polynomials orthogonal with respect to them. In particular, we are able to prove there PCM property of the analyzed distribution. The last Section, 4 presents some remarks and poses some open problems. 


\section{Multivariate Density}

We will study the following 3-dimensional density:

$$
\begin{aligned}
& f_{3 D}\left(x, y, z \mid \rho_{12}, \rho_{13}, \rho_{23}, q\right)=f_{N}(x \mid q) f_{N}(y \mid q) f_{N}(z \mid q) \\
& \times \frac{C_{3 D}\left(\rho_{12}^{2}\right)_{\infty}\left(\rho_{13}^{2}\right)_{\infty}\left(\rho_{23}^{2}\right)_{\infty}}{\prod_{i=0}^{\infty}\left(\omega_{q}\left(x, y \mid \rho_{12} q^{i}\right) \omega_{q}\left(x, z \mid \rho_{13} q^{i}\right) \omega_{q}\left(y, z \mid \rho_{23} q^{i}\right)\right)},
\end{aligned}
$$

where

$$
f_{N}(x \mid q)=\frac{(q)_{\infty} \sqrt{1-q} \sqrt{4-(1-q) x^{2}}}{2 \pi} \prod_{i=1}^{\infty} l_{q}\left(x \mid q^{i}\right)
$$

with

$$
\begin{gathered}
l_{q}(x \mid a)=(1+a)^{2}-(1-q) a x^{2}, \\
\omega_{q}(x, y \mid \rho)=\left(1-\rho^{2}\right)^{2}-(1-q) x y \rho\left(1+\rho^{2}\right)+(1-q) \rho^{2}\left(x^{2}+y^{2}\right),
\end{gathered}
$$

$|q|,\left|\rho_{12}\right|,\left|\rho_{13}\right|,\left|\rho_{23}\right|<1,|x|,|y|,|z| \leq 2 / \sqrt{1-q}$, and $C_{3 D}$ is a suitable constant. Symbol $(a)_{n}$ will be explained below.

Properties, alternative forms and ways of simulation of $f_{N}$ were presented in [4].

Ismail et al. in [3] proved rigorously that $f_{N}(x \mid q) \rightarrow \frac{1}{\sqrt{2 \pi}} \exp \left(-x^{2} / 2\right)$ as $q \rightarrow 1^{-}$.

2.1. Notation. We will use the following denotations

$$
\begin{aligned}
& {[0]_{q}=0,[n]_{q}=1+\ldots+q^{n-1}, n \geq 1,} \\
& {[n]_{q} !=\left\{\begin{array}{ccc}
1 & \text { if } & n=0 \\
\prod_{i=1}^{n}[i]_{q} & \text { if } & n \geq 1
\end{array}, S(q)=\left\{\begin{array}{ccc}
{\left[-\frac{2}{\sqrt{1-q}}, \frac{2}{\sqrt{1-q}}\right]} & \text { if } & |q|<1 \\
\mathbb{R} & \text { if } & q=1
\end{array},\right.\right.} \\
& {\left[\begin{array}{l}
n \\
k
\end{array}\right]_{q}=\left\{\begin{array}{ccc}
\frac{[n]_{q} !}{[k]_{q} ![n-k]_{q} !} & \text { if } & 0 \leq k \leq n \\
0 & \text { if } & \text { otherwise }
\end{array}\right.}
\end{aligned}
$$

and the so called $q$-Pochhammer symbol:

$$
(a)_{j}=\left\{\begin{array}{cll}
\prod_{k=1}^{j}\left(1-a q^{k-1}\right) & \text { if } & j \geq 1 \\
0 & \text { if } & j=0
\end{array} .\right.
$$

Let us note that $f_{3 D}$ is nonnegative on the cube $S(q)^{\times 3}$. Hence it can be viewed as a density.

It is obvious to notice that

$$
(q)_{n}=(1-q)^{n}[n]_{q} !
$$

For $q=0$ we have

$$
f_{3 D}\left(x, y, z \mid \rho_{12}, \rho_{13}, \rho_{23}, 0\right)=C_{3 D} \frac{\sqrt{\left(4-x^{2}\right)\left(4-y^{2}\right)\left(4-z^{2}\right)}}{\omega_{0}\left(x, y \mid \rho_{12}\right) \omega_{0}\left(x, z \mid \rho_{13}\right) \omega_{0}\left(y, z \mid \rho_{23}\right)},
$$

which is a variation of the multivariate generalization of Kesten-MacKay distribution (compare 12] formula 2.8 ), while by taking $q \rightarrow 1^{-}$we arrive at 3 dimensional Normal distribution with zero expectations and the following variance-covariance matrix :

$$
\left[\begin{array}{ccc}
\frac{1+r}{1-r} & \frac{\rho_{12}+\rho_{13} \rho_{23}}{1-r} & \frac{\rho_{13}+\rho_{12} \rho_{23}}{1-r} \\
\frac{\rho_{12}+\rho_{13} \rho_{23}}{1-r} & \frac{1+r}{1-r} & \frac{\rho_{23}+\rho_{12} \rho_{13}}{1-r} \\
\frac{\rho_{13}+\rho_{12} \rho_{23}}{1-r} & \frac{\rho_{23}+\rho_{12} \rho_{13}}{1-r} & \frac{1+r}{1-r}
\end{array}\right],
$$

where $r=\rho_{12} \rho_{13} \rho_{23}$. This fact follows (3.5), (3.6) and the properties of the function $f_{N}(x \mid q)$. 
2.2. Some auxiliary facts. The following facts were selected from [1], [6] and [5].

We will use the following families of orthogonal polynomials:

2.2.1. $q$-Hermite polynomials $\left\{H_{j}(x \mid q)\right\}_{j \geq-1}$. They satisfy the following recursive equations:

$$
H_{n+1}(x \mid q)=x H_{n}(x \mid q)-[n]_{q} H_{n-1}(x \mid q),
$$

with $H_{-1}(x \mid q)=0, H_{0}(x \mid q)=1$. They are orthogonal with respect to the density $f_{N}(x \mid q)$ i.e. we have:

$$
\int_{S(q)} H_{i}(x \mid q) H_{j}(x \mid q) f_{N}(x, q) d x=\delta_{i j}[i]_{q} !
$$

where $\delta_{i j}$ is the Kronecker's delta. In the sequel the following Poisson-Mehler summation formula will be of great help:

$$
\frac{\left(\rho^{2}\right)_{\infty}}{\prod_{i=0}^{\infty} \omega_{q}\left(x, y \mid \rho q^{i}\right)}=\sum_{j \geq 0} \frac{\rho^{j}}{[j]_{q} !} H_{j}(x \mid q) H_{j}(y \mid q)
$$

valid for all $|\rho|<1, x, y \in S(q)$.

Notice that we have useful formula:

$$
\sum_{j \geq 0} \frac{(\rho q)^{j}}{[j]_{q} !} H_{j}(x \mid q) H_{j}(y \mid q)=\frac{\omega_{q}(x, y \mid \rho)}{\left(1-\rho^{2}\right)\left(1-\rho^{2} q\right)} \sum_{j \geq 0} \frac{\rho^{j}}{[j]_{q} !} H_{j}(x \mid q) H_{j}(y \mid q),
$$

for all $|\rho|<1, x, y \in S(q)$, since $\frac{\left(q^{2} \rho^{2}\right)_{\infty}}{\prod_{i=0}^{\infty} \omega_{q}\left(x, y \mid \rho q^{i+1}\right)}=\frac{\omega_{q}(x, y \mid \rho)}{\left(1-\rho^{2}\right)\left(1-\rho^{2} q\right)} \frac{\left(\rho^{2}\right)_{\infty}}{\prod_{i=0}^{\infty} \omega_{q}\left(x, y \mid \rho q^{i}\right)}$.

Generalizations of (2.2) and their consequences are presented in [10].

The following formula will also be of help:

Lemma 1. We have

$$
\begin{gathered}
\int_{S(q)} H_{k}(x \mid q) H_{m}(x \mid q) H_{n}(x \mid q) f_{N}(x \mid q) d x= \\
\left\{\begin{array}{ccc}
k+m+n \text { is odd or } \\
0 & \text { if } & k+m<n \text { or } k+n<m \\
\left.\frac{[m]_{q} ![n]_{q} ![k]_{q} !}{2}\right]_{q} !\left[\frac{n+k-m}{2}\right]_{q} ! & \text { if } & \text { or } n+m<k \\
\left.\frac{m+n-m}{2}\right]_{q} !\left[\frac{m+k-n}{2}\right. & \text { otherwise }
\end{array} .\right.
\end{gathered}
$$

Proof. We use linearization formula 3.13 of [6]. It is obvious that the value of the integral is nonzero when $n+m-2 j=k$ for some $j \geq 0$, which means $m+n+k$ must be even and $n+m \geq k$. Note that formula must be symmetric with respect to $m, n, k$. Then this value is equal to $\left[\frac{n+m-k}{2}\right]_{q}\left[\frac{n+m}{2}\right]_{q}\left[\frac{n+m-k}{2}\right]_{q} ![k]_{q}$ ! which is equal to (2.4).

2.2.2. Al-Salam-Chihara polynomials $\left\{P_{n}(x \mid y, \rho, q)\right\}_{n \geq-1}$. They satisfy the following recursive equations:

$$
P_{n+1}(x \mid y, \rho, q)=\left(x-\rho y q^{n}\right) P_{n}(x \mid y, \rho, q)-\left(1-\rho^{2} q^{n-1}\right)[n]_{q} P_{n-1}(x \mid y, \rho, q),
$$


with $P_{-1}(x \mid y, \rho, q)=0, P_{0}(x \mid y, \rho, q)=1$. They turn out to be orthogonal with respect to the following densities:

$$
f_{C N}(x \mid y, \rho, q)=f_{N}(x \mid q) \frac{\left(\rho^{2}\right)_{\infty}}{\prod_{i=0}^{\infty} \omega_{q}\left(x, y \mid \rho q^{i}\right)} .
$$

That is we have:

$$
\int_{S(q)} P_{n}(x \mid y, \rho, q) P_{m}(x \mid y, \rho, q) f_{C N}(x \mid y, \rho, q) d x=\left\{\begin{array}{cl}
0 \quad \text { when } \quad n \neq m \\
\left(\rho^{2}\right)_{n}[n]_{q} ! & \text { when } n=m
\end{array}\right. \text {. }
$$

Moreover, we know that the densities $f_{C N}$ have the following interesting property (Chapman-Kolmogorov property):

$$
\int_{S(q)} f_{C N}\left(x \mid y, \rho_{1}, q\right) f_{C N}\left(y \mid z, \rho_{2}, q\right) d y=f_{C N}\left(x \mid z, \rho_{1} \rho_{2}, q\right) .
$$

Probabilistic aspects of Al-Salam-Chihara polynomials are presented in 9].

Notice that for $q \rightarrow 1^{-}$we have $\forall n \geq 0$ :

$$
P_{n}(x \mid y, \rho, q) \rightarrow H_{n}\left(\frac{x-\rho y}{\sqrt{1-\rho^{2}}}\right)\left(1-\rho^{2}\right)^{n / 2}
$$

and $H_{n}(x \mid q) \rightarrow H_{n}(x)$, where $H_{n}$ denotes the so-called probabilistic Hermite polynomials i.e. monic, orthogonal with respect to $\exp \left(-x^{2} / 2\right)$. Consequently we deduce that

$$
f_{C N}(x \mid y, \rho, q) \rightarrow \exp \left(-\frac{(x-\rho y)^{2}}{2\left(1-\rho^{2}\right)}\right) / \sqrt{2 \pi\left(1-\rho^{2}\right.},
$$

as $q \rightarrow 1^{-}$by (2.6).

Remark 1. Observe that making use of the density $f_{C N}$ as well as (2.2) we deduce that $f_{3 D}$ can be presented in one of the following two equivalent forms:

$$
f_{3 D}\left(x, y, z \mid \rho_{12}, \rho_{13}, \rho_{23}, q\right)=C_{3 D} f_{C N}\left(x \mid y, \rho_{12}, q\right) f_{C N}\left(y \mid z, \rho_{23}, q\right) f_{C N}\left(z \mid x, \rho_{13}, q\right) .
$$

and also that:

$$
\begin{gathered}
f_{3 D}\left(x, y, z \mid \rho_{12}, \rho_{13}, \rho_{23}, q\right)=C_{3 D} f_{N}(x \mid q) f_{N}(y \mid q) f_{N}(z \mid q) \\
\times \sum_{j, k, l \geq 0} \frac{\rho_{12}^{j} \rho_{23}^{k} \rho_{13}^{l}}{[j]_{q} ![k]_{q} ![l]_{q} !} H_{j}(x \mid q) H_{j}(y \mid q) H_{k}(y \mid q) H_{k}(z \mid q) H_{l}(x \mid q) H_{l}(z \mid q) .
\end{gathered}
$$

Hence we see that for $q \rightarrow 1^{-}$the density $f_{3 D}\left(x, y, z \mid \rho_{12}, \rho_{13}, \rho_{23}, q\right)$ tends to the density of Normal distribution with the variance-covariance matrix given in the introduction above.

2.2.3. Continuous q-ultraspherical (Rogers) polynomials. Name Rogers polynomials will be used for brevity. By Rogers polynomials it is meant (see e.g. 1]) family of polynomials $\left\{C_{n}(x \mid \beta, q)\right\}_{n \geq-1}$ defined by the following three term recurrence :

(2.10) $2 x\left(1-\beta q^{n}\right) C_{n}(x \mid \beta, q)=\left(1-q^{n+1}\right) C_{n+1}(x \mid \beta, q)+\left(1-\beta^{2} q^{n-1}\right) C_{n-1}(x \mid \beta, q)$, with $C_{-1}(x \mid \beta, q)=0, C_{0}(x \mid \beta, q)=1,|\beta|<1$. In fact we will need these polynomials modified in the following way:

$$
C_{n}(x \mid \beta, q)=\frac{(1-q)^{n / 2}(\beta)_{n}}{(q)_{n}} R_{n}\left(\frac{2 x}{\sqrt{1-q}} \mid \beta, q\right) .
$$


By inserting (2.11) into (2.10) and denoting $y=\frac{2 x}{\sqrt{1-q}}$, and canceling out $(\beta)_{n+1}(1-$ $q)^{(n+1) / 2} /(q)_{n}$, finally after little algebra, we end up with the following three term recurrence:

$$
y R_{n}(y \mid \beta, q)=R_{n+1}(y \mid \beta, q)+[n]_{q} \frac{\left(1-\beta^{2} q^{n-1}\right)}{\left(1-\beta q^{n-1}\right)\left(1-\beta q^{n}\right)} R_{n-1}(y \mid \beta, q),
$$

since $(\beta)_{n+1}=(\beta)_{n}\left(1-\beta q^{n}\right),(q)_{n}=(q)_{n-1}\left(1-q^{n}\right),[n]_{q}=\left(1-q^{n}\right) /(1-q)$. It is known also (see e.g. [6] (2.37) or in non modified form [1] (13.2.4)) that the measure that makes these polynomials orthogonal has the following density:

$$
f_{R}(x \mid \beta, q)=f_{N}(x \mid q) \frac{\left(\beta^{2}\right)_{\infty}}{(\beta)_{\infty}(\beta q)_{\infty} \prod_{i=0}^{\infty} l_{q}\left(x \mid \beta q^{i}\right)} .
$$

To simplify notation we will call distribution with the density $f_{R}$ the Rogers distribution. Notice that it is symmetric.

Note also that when $\beta=q$ then the equation (2.12) is simplified to

$$
y R_{n}(y \mid q, q)=R_{n+1}(y \mid q, q)+\frac{1}{1-q} R_{n-1}(y \mid q, q) .
$$

Hence $R_{n}(y \mid q, q)=U_{n}(y \sqrt{1-q} / 2) /(1-q)^{n / 2}$, where $U_{n}(x)$ denotes the Chebyshev polynomial of the second kind, since polynomials $\left\{U_{n}\right\}$ satisfy the following three term recurrence:

$$
2 x U_{n}(x)=U_{n+1}(x)+U_{n-1}(x),
$$

with $U_{-1}(x)=0$ and $U_{0}(x)=1$. Consequently one can see that

$$
f_{R}(x \mid q, q)=\frac{\sqrt{1-q} \sqrt{4-(1-q) x^{2}}}{2 \pi},
$$

$x \in S(q)$.

It is worth to mention the following formula :

$$
\sum_{i \geq 0} \frac{r^{i}}{[i]_{q} !} H_{i+k}(x \mid q) H_{i+m}(x \mid q)=W_{k, m}(x \mid r, q) \times \sum_{i \geq 0} \frac{r^{i}}{[i]_{q} !} H_{i}(x \mid q) H_{i}(x \mid q) .
$$

where $W_{k, m}(x \mid r, q)$ is given by the following formula:

$$
W_{k, m}(x \mid r, q)=\sum_{s=0}^{k} \frac{q^{\left(\begin{array}{c}
s \\
2
\end{array}\right)}(-r)^{s}(r)_{m+s}}{\left(r^{2}\right)_{m+s}} H_{k-s}(x \mid q) R_{m+s}(x \mid r, q) .
$$

From its definition it follows that $W_{k, m}(x \mid r, q)=W_{m, k}(x \mid r, q), k, m \geq 0$.

In particular taking into account (2.3) we have useful summation formula:

$$
\begin{aligned}
& \sum_{i \geq 0} \frac{(q r)^{i}}{[i]_{q} !} H_{i+k}(x \mid q) H_{i+m}(x \mid q) \\
= & W_{k, m}(x \mid r q, q) \frac{\omega_{q}(x, x \mid r)}{\left(1-r^{2}\right)\left(1-q r^{2}\right)} \times \sum_{i \geq 0} \frac{r^{i}}{[i]_{q} !} H_{i}(x \mid q) H_{i}(x \mid q) .
\end{aligned}
$$

(2.14) follows a formula in [7](Lemma $3 \mathrm{i})$ ), where we set $x=y$ and the following observation concerning relationship between polynomials $\left\{P_{n}(x \mid x, \rho, q)\right\}_{i \geq-1}$ and $\left\{R_{n}(x \mid \rho, q)\right\}_{i \geq-1}$ that:

$$
R_{n}(x \mid \rho, q)=P_{n}(x \mid x, \rho, q) /(\rho)_{n} .
$$




\section{MAin RESUlts}

\subsection{Marginals and moments.}

3.1.1. Marginal distributions. We will find $C_{3 D}$ as well as marginal densities first. We have the following result:

Theorem 1. Let us denote for simplicity $r=\rho_{12} \rho_{13} \rho_{23}$. Then

i) $C_{3 D}=1-r$.

ii) two-dimensional marginals depend on two parameters (except for $q$ ) in fact. In the case of $f_{Y Z}$ on $\rho_{23}$ and $\rho_{12} \rho_{13}$ only.

$$
\begin{gathered}
f_{Y Z}\left(y, z \mid \rho_{12}, \rho_{13}, \rho_{23}, q\right)=\int_{S(q)} f_{3 D}\left(x, y, z \mid \rho_{12}, \rho_{13}, \rho_{23}, q\right) d x= \\
(1-r) f_{N}(y \mid q) f_{N}(z \mid q) \frac{\left(\rho_{23}^{2}\right)_{\infty}\left(\rho_{12}^{2} \rho_{13}^{2}\right)_{\infty}}{\prod_{i=0}^{\infty} \omega\left(y, z \mid \rho_{23} q^{i}\right) \omega\left(y, z \mid \rho_{12} \rho_{13} q^{i}\right)} \\
=(1-r) f_{C N}\left(y \mid z, \rho_{23}, q\right) f_{C N}\left(z \mid y, \rho_{12} \rho_{13}, q\right)
\end{gathered}
$$

and similarly for $f_{X Z}$, and $f_{X Y}$.

iii) Marginal one dimensional densities $\int_{S(q)} \int_{S(q)} f_{3 D}\left(x, y, z \mid \rho_{12}, \rho_{13}, \rho_{23}, q\right) d x d y=$ $f_{Z}\left(z \mid \rho_{12}, \rho_{13}, \rho_{23}, q\right)$ depend on the product $r=\rho_{12} \rho_{23} \rho_{13}$ only. More over we have $f_{Z}\left(z \mid \rho_{12}, \rho_{13}, \rho_{23}, q\right)=f_{R}(z \mid r, q)$, where $f_{R}$ is a Rogers distribution given by (2.13).

Proof. We will use representation (2.8) and the property (2.7). First, let us calculate the integral:

$$
\begin{gathered}
f_{Y Z}\left(y, z \mid \rho_{12}, \rho_{13}, \rho_{23}, q\right)=\int_{S(q)} f_{3 D}\left(x, y, z \mid \rho_{12}, \rho_{13}, \rho_{23}, q\right) d x= \\
C_{3 D} f_{C N}\left(y \mid z, \rho_{23}, q\right) \int_{S(q)} f_{C N}\left(x \mid y, \rho_{12}, q\right) f_{C N}\left(z \mid x, \rho_{13}, q\right) d x \\
=C_{3 D} f_{C N}\left(y \mid z, \rho_{23}, q\right) f_{C D}\left(z \mid y, \rho_{12} \rho_{13}, q\right) \\
=C_{3 D} f_{N}(y \mid q) f_{N}(z \mid q) \frac{\left(\rho_{23}^{2}\right)_{\infty}\left(\rho_{12}^{2} \rho_{13}^{2}\right)_{\infty}}{\prod_{i=0}^{\infty} \omega_{q}\left(y, z \mid \rho_{23} q^{i}\right) \omega_{q}\left(y, z \mid \rho_{12} \rho_{13} q^{i}\right)} .
\end{gathered}
$$

Now let us calculate integral

$$
\begin{gathered}
f_{Z}\left(z \mid \rho_{12}, \rho_{13}, \rho_{23}, q\right)=\int_{S(q)} \int_{S(q)} f_{3 D}\left(x, y, z \mid \rho_{12}, \rho_{13}, \rho_{23}, q\right) d x d y= \\
C_{3 D} f_{N}(z \mid q) \int_{S(q)} \frac{\left(\rho_{23}^{2}\right)_{\infty}\left(\rho_{12}^{2} \rho_{13}^{2}\right)_{\infty}}{\prod_{i=0}^{\infty} \omega_{q}\left(y, z \mid \rho_{23} q^{i}\right) \omega_{q}\left(y, z \mid \rho_{12} \rho_{33} q^{i}\right)} f_{N}(y \mid q) d y \\
=C_{3 D} f_{N}(z \mid q) \int_{S(q)} f_{N}(y \mid q)\left(\sum_{k, j \geq 0} \frac{\left(\rho_{12} \rho_{13}\right)^{j} \rho_{23}^{k}}{[j]_{q} ![k]_{q} !} H_{j}(z \mid q) H_{j}(y \mid q) H_{k}(y \mid q) H_{k}(z \mid q)\right) d y \\
=C_{3 D} f_{N}(z \mid q) \sum_{j \geq 0} \frac{\left(\rho_{12} \rho_{13} \rho_{23}\right)^{j}}{[j]_{q} !} H_{j}^{2}(z \mid q) .
\end{gathered}
$$


Now notice that using (2.2) and noticing that $\omega_{q}(x, x \mid r)=(1-r)^{2} l_{q}(x \mid r)$

$$
\begin{aligned}
\sum_{j \geq 0} \frac{(r)^{j}}{[j]_{q} !} H_{j}^{2}(z \mid q)= & \frac{\left(r^{2}\right)_{\infty}}{\prod_{i=0}^{\infty}\left(\omega_{q}\left(z, z \mid r q^{i}\right)\right.}=\frac{\left(r^{2}\right)_{\infty}}{\prod_{i=0}^{\infty}\left(1-r q^{i}\right)^{2} l_{q}\left(z \mid r q^{i}\right)} \\
& \frac{\left(r^{2}\right)_{\infty}}{(r)_{\infty}^{2} \prod_{i=0}^{\infty} l\left(z \mid r q^{i}\right)} .
\end{aligned}
$$

Comparing this with (2.13) we see that

$$
f_{Z}\left(z \mid \rho_{12}, \rho_{13}, \rho_{23}, q\right)=C_{3 D} f_{R}(z \mid r, q) /(1-r),
$$

since $(r)_{\infty}=(1-r)(r q)_{\infty}$. Now since $f_{R}$ is the density we deduce that $C_{3 D}=$ $1-\rho_{12} \rho_{13} \rho_{23}=1-r$.

Proposition 1. The densities $f_{Z}\left(z \mid \rho_{12}, \rho_{13}, \rho_{23}, q\right), f_{Y}$ and $f_{X}$ are of one of the following equivalent forms:

$$
\begin{gathered}
(1-r) f_{N}(z \mid q) \sum_{j \geq 0} \frac{r^{j}}{[j]_{q} !} H_{j}^{2}(z \mid q)=(1-r) f_{C N}(z \mid z, r, q) \\
=(1-r) f_{N}(z \mid q) \frac{\left(r^{2}\right)_{\infty}}{(r)_{\infty}^{2} \prod_{i=0}^{\infty} l_{q}\left(z \mid r q^{i}\right)} \\
=(1-r) f_{N}(z \mid q) \sum_{k=0}^{\infty} \frac{r^{k}}{[k]_{q} !(r)_{k+1}} H_{2 k}(x \mid q) \\
=\frac{2(1+r) \sqrt{4-(1-q) z^{2}}(q)_{\infty}\left(r^{2} q\right)_{\infty}}{\pi l_{q}(z \mid r)(r q)_{\infty}^{2}} \prod_{j=1}^{\infty} \frac{l_{q}\left(z \mid q^{j}\right)}{l_{q}\left(z \mid r q^{j}\right)}
\end{gathered}
$$

Proof. Notice that we have:

$$
\sum_{j \geq 0} \frac{r^{j}}{[j]_{q} !} H_{j}^{2}(z \mid q)=\sum_{j \geq 0} \frac{r^{j}}{[j]_{q} !} \sum_{k=0}^{j} \frac{\left([j]_{q} !\right)^{2}}{\left([k]_{q} !\right)^{2}[j-k]_{q} !} H_{2 k}(x \mid q),
$$

since $H_{j}^{2}(x \mid q)=\sum_{k=0}^{j} \frac{\left([j]_{q} !\right)^{2}}{\left([k]_{q} !\right)^{2}[j-k]_{q} !} H_{2 k}(x \mid q)$ by [6] (3.13). Hence

$$
\begin{gathered}
f_{Z}\left(z \mid \rho_{12}, \rho_{13}, \rho_{23}, q\right)=(1-r) f_{N}(z \mid q) \sum_{k=0}^{\infty} \frac{r^{k}}{[k]_{q} !} H_{2 k}(x \mid q) \sum_{j \geq k} r^{j-k}\left[\begin{array}{l}
j \\
k
\end{array}\right]_{q} \\
=(1-r) f_{N}(z \mid q) \sum_{k=0}^{\infty} \frac{r^{k}}{[k]_{q} !(r)_{k+1}} H_{2 k}(x \mid q),
\end{gathered}
$$

because $\sum_{m \geq 0} r^{m}\left[\begin{array}{c}m+k \\ k\end{array}\right]_{q}=1 /(r)_{k+1}$.

To get third, multiplicative form we argue as follows

$$
\begin{gathered}
f_{Z}(z \mid r, q)=(1-r) f_{N}(z \mid q) \sum_{j \geq 0} \frac{r^{j}}{[j]_{q} !} H_{j}^{2}(z \mid q) \\
=\frac{2\left(1-r^{2}\right) \sqrt{4-(1-q) z^{2}}(q)_{\infty}\left(r^{2}\right)_{\infty}}{\pi l_{q}(z \mid r)(r)_{\infty}^{2}} \prod_{j=1}^{\infty} \frac{l_{q}\left(z \mid q^{j}\right)}{l_{q}\left(z \mid r q^{j}\right)} \\
=\frac{2 \sqrt{4-(1-q) z^{2}}(1+r)(q)_{\infty}\left(r^{2} q\right)_{\infty}}{\pi l_{q}(z \mid r)(r q)_{\infty}^{2}} \prod_{j=1}^{\infty} \frac{l_{q}\left(z \mid q^{j}\right)}{l_{q}\left(z \mid r q^{j}\right)} .
\end{gathered}
$$


Proposition 2. Polynomials that are orthogonal with respect to $f_{R}$ belong to the family $\left\{w_{n}(x \mid r, q)\right\}_{n>-1}$ of monic Rogers polynomials satisfying three term recurrence given by (2.12) with $\beta=r$. Notice that $w_{n}(x \mid r, q)=P_{n}(x \mid x, r, q) /(r)_{n}$. Moreover we have

$$
\int_{S(q)} w_{n}(x \mid r, q) w_{m}(x \mid r, q) f_{R}(x \mid r, q)=\left\{\begin{array}{cll}
0 & \text { if } & n \neq m \\
{[n]_{q} ! \frac{(1-r)\left(r^{2}\right)_{n}}{(r)_{n}(r)_{n+1}}} & \text { if } & m=n
\end{array} .\right.
$$

Proof. Consider (2.12) and apply formula (2.2.18) of [1].

3.1.2. Moments. Now let us calculate some moments of these variables. Notice that since function $l$ depends on $z^{2}$ we deduce that $f_{Z}(z \mid r, q)$ is symmetric in $z$, consequently all odd moments of $Z$ are equal to zero.

Further, we have the following lemma:

Lemma 2. i) Suppose $Z \sim f_{Z}(z \mid r, q)$, with $|r|,|q|<1, z \in S(q)$, then

In particular

$$
E H_{2 n}(Z \mid q)=\frac{r^{n}[2 n]_{q} !}{[n]_{q} !(r q)_{n}} \text {. }
$$

$$
E Z=0, \quad \operatorname{var}(Z)=E Z^{2}=\frac{1+r}{1-r q} .
$$

ii) Let $(Y, Z) \sim f_{Y Z}\left(y, z \mid \rho_{12}, \rho_{13}, \rho_{23}, q\right)$, then

$$
\begin{gathered}
E H_{m}(Y \mid q) H_{n}(Z \mid q)=\sum_{s \geq \max (n, m)} \frac{1}{\left[\frac{s-m}{2}\right]_{q} !\left[\frac{s-n}{2}\right]_{q} !} \\
\times \sum_{k=\frac{s}{2}-\min \left(\frac{n}{2}, \frac{m}{2}\right)}^{\min \left(\frac{n}{2}, \frac{m}{2}\right)+\frac{s}{2}} \rho_{23}^{k}\left(\rho_{12} \rho_{13}\right)^{s-k}\left[\begin{array}{c}
m \\
k-\frac{s-m}{2}
\end{array}\right]_{q}\left[\begin{array}{c}
n \\
\left.k-\frac{s-n}{2}\right]_{q}
\end{array}[k]_{q} ![s-k]_{q} !\right.
\end{gathered}
$$

In particular

$$
\operatorname{cov}(Y, Z)=\frac{\left(\rho_{23}+\rho_{12} \rho_{13}\right)}{(1-r q)} .
$$

Proof. i) Let us calculate $\int_{S(q)} H_{2 n}(z \mid q) f_{Z}(z \mid r, q) d z$. Using (3.3) we have

$$
\int_{S(q)} H_{2 n}(z \mid q) f_{Z}(z \mid r, q) d z=(1-r) \sum_{k=0}^{\infty} \frac{r^{k}}{[k]_{q} !(r)_{k+1}} \int_{S(q)} H_{2 n}(z \mid q) H_{2 k}(z \mid q) f_{N}(z \mid q) d z .
$$

Hence

$$
\int_{S(q)} H_{2 n}(z \mid q) f_{Z}(z \mid r, q) d z=(1-r) \frac{r^{n}[2 n]_{q} !}{[n]_{q} !(r)_{k+1}} .
$$

by (2.1). Recall also that $\frac{(1-r)}{(r)_{k+1}}=\frac{1}{(r q)_{k}}$. For $n=1$ we get $E H_{2}(Z \mid q)=r \frac{1+q}{1-r q}$. Now recall that $H_{2}(x \mid q)=x^{2}-1$.

ii) We have, denoting $r=\rho_{12} \rho_{13} \rho_{23}$ :

$$
\begin{gathered}
E H_{m}(Y \mid q) H_{n}(Z \mid q)=(1-r) \sum_{j, k \geq 0} \frac{\rho_{23}^{k}\left(\rho_{12} \rho_{13}\right)^{j}}{(q)_{j}(q)_{k}} \\
\times \int_{S(q)} H_{m}(y \mid q) H_{k}(y \mid q) H_{j}(y \mid q) f_{N}(y \mid q) d y \int_{S(q)} H_{n}(z \mid q) H_{k}(z \mid q) H_{j}(z \mid q) f_{N}(z \mid q) d z .
\end{gathered}
$$


Now we apply Lemma 1 and we have further:

$$
\begin{gathered}
E H_{m}(Y \mid q) H_{n}(Z \mid q)=(1-r) \\
\times \sum_{j, k \geq 0} \rho_{23}^{k}\left(\rho_{12} \rho_{13}\right)^{j} \frac{[n]_{q} ![m]_{q} ![j]_{q} ![k]_{q} !}{\left[\frac{n+j-k}{2}\right]_{q} !\left[\frac{m+j-k}{2}\right]_{q} !\left[\frac{n+k-j}{2}\right]_{q} !\left[\frac{m+k-j}{2}\right]_{q} !\left[\frac{j+k-m}{2}\right]_{q} !\left[\frac{j+k-n}{2}\right]_{q} !} \\
=(1-r) \sum_{s \geq \max (n, m)} \frac{1}{\left[\frac{s-m}{2}\right]_{q} !\left[\frac{s-n}{2}\right]_{q} !} \times \\
\sum_{k=\frac{s}{2}-\min \left(\frac{n}{2}, \frac{m}{2}\right)}^{\min \left(\frac{n}{2}, \frac{m}{2}\right)+\frac{s}{2}} \rho_{23}^{k}\left(\rho_{12} \rho_{13}\right)^{s-k}\left[\begin{array}{c}
m \\
k-\frac{s-m}{2}
\end{array}\right]_{q}\left[\begin{array}{c}
n \\
k-\frac{s-n}{2}
\end{array}\right]_{q}[k]_{q} ![s-k]_{q} !
\end{gathered}
$$

For $m=n=1$ we see that $s+m$ as well as $s+n$ have to be even, hence $s$ has to be odd. Thus, since $H_{1}(x \mid q)=x$, we get

$$
\begin{gathered}
\operatorname{cov}(Y, Z)=E Y Z=(1-r) \sum_{t=0}^{\infty} \frac{1}{[t]_{q} ![t]_{q} !} \sum_{k=t}^{t+1} \rho_{23}^{k}\left(\rho_{12} \rho_{13}\right)^{2 t+1-k}[k]_{q} ![2 t+1-k]_{q} != \\
(1-r) \sum_{t=0}^{\infty} \frac{1}{[t]_{q} ![t]_{q} !}\left(\rho_{23}^{t}\left(\rho_{12} \rho_{13}\right)^{t+1}[t]_{q} ![t+1]_{q} !+\rho_{23}^{t+1}\left(\rho_{12} \rho_{13}\right)^{t}[t]_{q} ![t+1]_{q} !\right)= \\
(1-r)\left(\rho_{23}+\rho_{12} \rho_{13}\right) \sum_{t=0}^{\infty} r^{t}[t+1]_{q}=\frac{(1-r)}{1-q}\left(\rho_{23}+\rho_{12} \rho_{13}\right)\left(\frac{1}{1-r}-\frac{q}{1-r q}\right) \\
=\frac{\left(\rho_{23}+\rho_{12} \rho_{13}\right)}{1-r q}
\end{gathered}
$$

\subsection{Conditional distributions and conditional moments.}

3.2.1. Conditional distributions. As far as conditional distributions are concerned, we have the following simple result

Proposition 3. Following formulae (2.8) and (2.6) we have:

$$
\begin{gathered}
X \mid(Y=y, Z=z) \sim f_{X \mid Y=y, Z=z}\left(x \mid y, z, \rho_{12}, \rho_{13}, \rho_{23}, q\right) \\
=f_{N}(x \mid q) \frac{\left(\rho_{12}^{2}, \rho_{13}^{2}\right)_{\infty} \prod_{i=0}^{\infty} \omega\left(y, z \mid \rho_{12} \rho_{13} q^{i}\right)}{\left(\rho_{12}^{2} \rho_{13}^{2}\right)_{\infty} \prod_{i=0}^{\infty}\left(\omega_{q}\left(x, y \mid \rho_{12} q^{i}\right) \omega_{q}\left(x, z \mid \rho_{13} q^{i}\right)\right.} \\
=\frac{f_{C N}\left(x \mid y, \rho_{12}, q\right) f_{C N}\left(z \mid x, \rho_{13}, q\right)}{f_{C N}\left(z \mid y, \rho_{12} \rho_{13}, q\right)} \\
Y, Z \mid X=x \sim f_{Y, Z \mid X=x}\left(y, z \mid x, \rho_{12}, \rho_{13}, \rho_{23}, q\right) \\
=\frac{f_{C N}\left(x \mid y, \rho_{12}, q\right) f_{C N}\left(y \mid z, \rho_{23}, q\right) f_{C N}\left(z \mid x, \rho_{13}, q\right)}{f_{C N}(x \mid x, r, q)} .
\end{gathered}
$$

3.2.2. Conditional moments. It has to be noted in this sections that whenever one considers conditional expectation, then all equalities are considered almost surely with respect to the distributions of the conditioning random variable(s).

Remark 2. Following observation contained in the formula (3.7), the results presented in [7] and then in [5] we deduce that conditional distribution of $X \mid(Y=$ 
$y, Z=z)$ is the distribution that makes Askey-Wilson polynomials with complex but conjugate parameters orthogonal. More precisely, we take:

$$
\begin{aligned}
a & =\frac{\sqrt{1-q}}{2} \rho_{1}\left(y-i \sqrt{\frac{4}{1-q}-y^{2}}\right), \\
b & =\frac{\sqrt{1-q}}{2} \rho_{1}\left(y+i \sqrt{\frac{4}{1-q}-y^{2}}\right), \\
c & =\frac{\sqrt{1-q}}{2} \rho_{2}\left(z-i \sqrt{\frac{4}{1-q}-z^{2}}\right), \\
d & =\frac{\sqrt{1-q}}{2} \rho_{2}\left(z+i \sqrt{\frac{4}{1-q}-z^{2}}\right) .
\end{aligned}
$$

From 7] (2.15) and [5] (4.9) we deduce that the conditional expectations $E\left(H_{n}(X \mid q) \mid Y, Z\right)$ are polynomials in $Z$ and $Y$ of order $n$. Following these results we have two equivalent forms of this function: (7] (3.2) and [7] Lemma 3ii)):

Proposition 4. $E\left(H_{n}(X \mid q) \mid Y=y, Z=z\right)$ has one of the following equivalent form:

$$
\begin{gathered}
E\left(H_{n}(X \mid q) \mid Y=y, Z=z\right) \\
=\sum_{s=0}^{n}\left[\begin{array}{l}
n \\
s
\end{array}\right]_{q} \rho_{12}^{n-s} \rho_{13}^{s}\left(\rho_{12}^{2}\right)_{s} H_{n-s}(y \mid q) P_{s}\left(z \mid y, \rho_{12} \rho_{13}, q\right) /\left(\rho_{12}^{2} \rho_{13}^{2}\right)_{s},
\end{gathered}
$$

where $\left\{P_{s}\left(z \mid y, \rho_{1} \rho_{2}, q\right)\right\}_{s \geq-1}$ constitute the so called Al-Salam-Chihara polynomials given by (2.5).

ii)

$$
\begin{gathered}
E\left(H_{n}(X \mid q) \mid Y=y, Z=z\right)=\frac{1}{\left(\rho_{12}^{2} \rho_{13}^{2}\right)_{n}} \sum_{k=0}^{\lfloor n / 2\rfloor}(-1)^{k} q^{\left(\begin{array}{c}
k \\
2
\end{array}\right)}\left[\begin{array}{c}
n \\
2 k
\end{array}\right]_{q}\left[\begin{array}{c}
2 k \\
k
\end{array}\right]_{q}[k]_{q} ! \rho_{13}^{2 k} \rho_{12}^{2 k} \\
\times\left(\rho_{12}^{2}, \rho_{13}^{2}\right)_{k} \sum_{j=0}^{n-2 k}\left[\begin{array}{c}
n-2 k \\
j
\end{array}\right]_{q}\left(\rho_{12}^{2} q^{k}\right)_{j}\left(\rho_{13}^{2} q^{k}\right)_{n-2 k-j} \rho_{12}^{n-2 k-j} \rho_{13}^{j} H_{j}(z \mid q) H_{n-2 k-j}(y \mid q) .
\end{gathered}
$$

iii) $E\left(P_{n}\left(X \mid y, \rho_{12}, q\right) \mid Y=y, Z=z\right)=\frac{\rho_{13}^{n}\left(\rho_{12}^{2}\right)_{n}}{\left(\rho_{12}^{2} \rho_{13}^{2}\right)_{n}} P_{n}\left(z \mid y, \rho_{12} \rho_{13}, q\right)$.

In particular we have

$$
E(X \mid Y=y, Z=z)=\frac{y \rho_{12}\left(1-\rho_{13}^{2}\right)+z \rho_{13}\left(1-\rho_{12}^{2}\right)}{1-\rho_{12}^{2} \rho_{13}^{2}} .
$$

Proof. Following Remark 3 we recall that following [7](2.15), properties of $f_{X \mid Y, Z}\left(x \mid y, z, \rho_{12}, \rho_{13}, \rho_{23}, q\right)$ are basically known. In particular following (3.2-3.3) of [7] we get ii). Further following Lemma 3 ii) of [7] we get i). Finally following Thm. 4.1iii) of [5] we get iii). Now taking either i) or iii) and $n=1$ and $H_{1}(x \mid q)=x$, $P_{1}(x \mid y, \rho, q)=x-\rho y$ we get 3.15

We also have similar result concerning one dimensional conditional moments: 
Theorem 2. One dimensional conditional moments say $E\left(H_{n}(Y \mid q) \mid Z=z\right)$ are polynomials of order not exceeding $n$ in $Z$. More precisely for $n=2 m+1$ we have $E\left(H_{2 m+1}(Y \mid q) \mid Z=z\right)=\sum_{s=0}^{m}\left[\begin{array}{c}2 m+1 \\ s\end{array}\right]_{q}\left(\rho_{23}^{2 m+1-s}+\left(\rho_{12} \rho_{13}\right)^{2 m+1-s}\right) W_{s, 2 m+1-s}(z \mid r, q)$, and for $n=2 m, m \geq 1$ we have:

$$
\begin{gathered}
E\left(H_{2 m}(Y \mid q) \mid Z=z\right)=\left[\begin{array}{c}
2 m \\
m
\end{array}\right]_{q} r^{m} W_{m, m}(z \mid r, q) \\
+\sum_{s=0}^{m-1}\left[\begin{array}{c}
2 m \\
s
\end{array}\right]_{q}\left(\rho_{23}^{2 m-s}+\left(\rho_{12} \rho_{13}\right)^{2 m-s}\right) W_{s, 2 m-s}(z \mid r, q),
\end{gathered}
$$

where polynomials $\left\{W_{m, k}(z \mid r, q)\right\}_{m, k \geq 0}$ are given by (2.15).

In particular we have:

$$
E(Y \mid Z=z)=\frac{\left(\rho_{23}+\rho_{12} \rho_{13}\right)}{(1+r)} z
$$

and

$E\left(Y^{2} \mid Z=z\right)=\frac{\left(\rho_{23}^{2}+\rho_{12}^{2} \rho_{13}^{2}\right)(1-q r)+r(1-r)(1+q)}{(1+r)\left(1-q r^{2}\right)} z^{2}+\frac{1+r^{2}-\rho_{23}^{2}-\rho_{12}^{2} \rho_{13}^{2}}{\left(1-q r^{2}\right)}$.

Proof. We start with formula (2.4) that we apply to expression for $E\left(H_{n}(Y \mid q) \mid Z=\right.$ $z$ ) that is equal to

$$
\begin{gathered}
E\left(H_{n}(Y \mid q) \mid Z=z\right) f_{R}(z \mid r, q)=(1-r) f_{N}(z \mid q) \sum_{j, i \geq 0} \frac{\rho_{23}^{i}\left(\rho_{12} \rho_{13}\right)^{j}}{[i]_{q} ![j]_{q} !} H_{i}(z \mid q) H_{j}(z \mid q) \\
\times \int_{S(q)} H_{n}(y \mid q) H_{i}(y \mid q) H_{j}(y \mid q) f_{N}(y \mid q) d y= \\
(1-r) f_{N}(z \mid q) \sum_{j, i \geq 0} \frac{\rho_{23}^{i}\left(\rho_{12} \rho_{13}\right)^{j}}{[i]_{q} ![j]_{q} !} H_{i}(z \mid q) H_{j}(z \mid q) \frac{[j]_{q} ![i]_{q} ![n]_{q} !}{\left[\frac{i+j-n}{2}\right]_{q} !\left[\frac{i+n-j}{2}\right]_{q} !\left[\frac{j+n-i}{2}\right]_{q} !}= \\
(1-r) f_{N}(z \mid q) \sum_{j, i \geq 0} \frac{\rho_{23}^{i}\left(\rho_{12} \rho_{13}\right)^{j}[n]_{q} !}{\left[\frac{i+j-n}{2}\right]_{q} !\left[\frac{i+n-j}{2}\right]_{q} !\left[\frac{j+n-i}{2}\right]_{q} !} H_{i}(z \mid q) H_{j}(z \mid q) .
\end{gathered}
$$

Now if $n$ is odd, say of the form $n=2 m+1, m \geq 0$ we notice that all three numbers $\frac{i+j-n}{2}, \frac{i+n-j}{2}, \frac{j+n-i}{2}$ are simultaneously nonnegative and integer for $j=$ $i+1, i+3, \ldots, i+(2 m+1)$ and $i=j+1, j+3, \ldots, j+(2 m+1)$. Then these numbers are equal to respectively $(i-m, m, m+1),(i-m+1, m-1, m+2)$, $\ldots,(i, 0,2 m+1)(j-m, m, m+1),(j-m+1, m-1, m+2), \ldots,(j, 0,2 m+1)$. If say $j=i+2(m-s)+1$ these numbers are equal to $(i-s, s, 2 m+1-s)$ we have the sum (keeping in mind that $r=\rho_{12} \rho_{13} \rho_{23}$ ):

$$
\begin{gathered}
(1-r) f_{N}(z \mid q) \sum_{i \geq s} \frac{\rho_{23}^{i-s}[2 m+1]_{q} !\left(\rho_{12} \rho_{13}\right)^{i+2(m-s)+1}}{[i-s]_{q} ![s]_{q} ![2 m+1-s]_{q} !} H_{i}(z \mid q) H_{i+2(m-s)+1}(z \mid q)= \\
(1-r) f_{N}(z \mid q)\left(\rho_{12} \rho_{13}\right)^{2 m-s+1}\left[\begin{array}{c}
2 m+1 \\
s
\end{array}\right]_{q} \sum_{l \geq 0} \frac{r_{23}^{l}}{[l]_{q} !} H_{l+s}(z \mid q) H_{l+(2 m-s+1)}(z \mid q) .
\end{gathered}
$$


Now recall (2.14) that states that $\sum_{l \geq 0} \frac{r_{23}^{l}}{[l]_{q} !} H_{l+s}(z \mid q) H_{l+(2 m-s+1)}(z \mid q)$ is equal to some polynomial in $z$ of order $s+(2 m-s)+1=2 m+1$ times $\sum_{l \geq 0} \frac{r_{23}^{l}}{[l]_{q} !} H_{l}^{2}(z \mid q)$. But $(1-r) f_{N}(z \mid q) \sum_{l \geq 0} \frac{r_{23}^{l}}{[l]_{q} !} H_{l}^{2}(z \mid q)$ is the marginal density of $Z$. Thus we see that for $s=0, \ldots, m$ we get linear combination of some (depending on $s$ ) polynomials of order $2 m+1$ in $z$ with coefficients equal to $\left[\begin{array}{c}2 m-1 \\ s\end{array}\right]_{q}\left(\rho_{12} \rho_{13}\right)^{2 m-s+1}$ times the density of $f_{Z}(z \mid r, q)$. If say $i=j+2(m-s)+1$ then we have similar situation. The only difference lies in fact that this time linear combination is with $\left[\begin{array}{c}2 m+1 \\ s\end{array}\right]_{q}\left(\rho_{23}\right)^{2 m-s+1}$.

Similar situation is when $n=2 m, m \geq 1$. Then numbers $\frac{i+j-n}{2}, \frac{i+n-j}{2}, \frac{j+n-i}{2}$ are simultaneously nonnegative and integer for $i=j, j+2, \ldots, j+2 m$ and $j=$ $i+2, \ldots i+2 m$. Altogether we will have $2 m+1$ summands. Again each of them will be equal to some polynomial of order $2 m$ in $z$ times $\sum_{l \geq 0} \frac{r_{23}^{l}}{[l]_{q} !} H_{l}^{2}(z \mid q)$ with different (depending on if $j=i+2 s$ or $i=j+2 s$ ) coefficients of linear combination.

Now let us apply these ideas to cases $k=1$ and $k=2$. Recall that $H_{1}(x \mid q)=x$ and $H_{2}(x \mid q)=x^{2}-1, W_{1,0}(x \mid r, q)=\frac{x}{1+r}, W_{1,1}(x \mid r, q)=x^{2} \frac{1-r}{(1+r)\left(1-q r^{2}\right)}+\frac{r}{\left(1-q r^{2}\right)}$, $W_{2,0}(x \mid r, q)=x^{2} \frac{1-q r}{(1+r)\left(1-q r^{2}\right)}-\frac{1}{1-q r^{2}}$. Hence $E(Y \mid Z)$ is indeed given in ii). iii) we get likewise.

Corollary 1. $\forall n, m \geq 0: E\left(H_{n}(X \mid q) H_{m}(Y \mid q) \mid Z\right)$ is a polynomial of order at most $n+m$ of the conditioning random variable $Z$.

In particular we have:

$$
\begin{aligned}
(3.18) E(X Y \mid Z= & z)=z^{2} \frac{\rho_{12}\left(\rho_{13}^{2}+\rho_{23}^{2}\right)(1-q r)+(1-r)\left(\rho_{13} \rho_{23}+q r \rho_{12}\right)}{(1+r)\left(1-q r^{2}\right)} \\
& +\frac{\rho_{12}\left(1-\rho_{13}^{2}\right)\left(1-\rho_{23}^{2}\right)}{\left(1-q r^{2}\right)} .
\end{aligned}
$$

Proof. By the tower property of conditional expectation we have

$$
E\left(H_{n}(X \mid q) H_{m}(Y \mid q) \mid Z=z\right)=E\left(H_{m}(Y \mid q) E\left(H_{n}(X \mid q) \mid(Y, Z)\right) \mid Z=z\right)
$$

Using an assertion of Proposition 4 we deduce that $E\left(H_{n}(X \mid q) \mid(Y=y, Z=z)\right)$ is a polynomial of order $n$ in $y$ and $z$. Hence $H_{m}(Y \mid q) E\left(H_{n}(X \mid q) \mid(Y=y, Z=z)\right)$ is a polynomial of order $n+m$ in $y$ and $n$ in $z$ but together of order $n+m$ in both variables. It follows formulae (3.13) or (3.14). Hence, indeed $E\left(H_{n}(X \mid q) H_{m}(Y \mid q) \mid=z\right)$ is a polynomial of order $n+m$ in $z$.

To get (3.18) we combine (3.15), (3.16) and (3.17) and then use Mathematica.

\section{REMARKS AND OPEN PROBLEMS}

1. The most important remark seems to be an observation of the fact that all conditional moments of order say $n$ are also polynomials of order $n$ in the conditioning random variables. Among three-dimensional distributions having similar property are the Gaussian distributions. Are there any others except these two mentioned?

2. It could of interest to consider two extremal cases, i.e. $q=0$ and $q \rightarrow 1^{-}$.

2a. Following (2.12), for $q=0$ we get: $w_{-1}(x \mid r, 0)=0, w_{0}(x \mid r, 0)=1$, $w_{1}(x \mid r, 0)=x, w_{2}(x \mid r, 0)=x^{2}-1$ and further for $n \geq 2$ the recursion (2.12) becomes the following:

$$
w_{n+1}(x \mid r, 0)=x w_{n}(x \mid r, 0)-w_{n-1}(x \mid r, 0)
$$


which is the recurrence satisfied by the Chebyshev polynomials. Consequently, we deduce that for $n \geq 1$ we have:

$$
w_{n}(x \mid r, 0)=U_{n}(x / 2)-(2-r) U_{n-2}(x / 2) .
$$

With little of algebra applied to (3.1) with $q=0$ we get the following density of one dimensional distribution:

$$
f_{X}(x \mid r, 0)=\frac{(1+r) \sqrt{4-x^{2}}}{2 \pi\left((1+r)^{2}-r x^{2}\right)}
$$

that is Kesten-McKay distribution.

$2 \mathrm{~b}$. On the other hand again following (2.12) for $q \rightarrow 1^{-}$we get: $w_{-1}(x \mid r, 1)=0$, $w_{0}(x \mid r, 1)=1$, and for $n \geq 0$

$$
w_{n+1}(x \mid r, 1)=x w_{n}(x \mid r, 1)-\frac{1+r}{1-r} n w_{n-1}(x \mid r, 1) .
$$

Recall also that the so called probabilistic Hermite polynomials $\left\{H_{n}(x)\right\}_{n \geq-1}$ i.e. orthogonal with respect to $\exp \left(-x^{2} / 2\right) / \sqrt{2}$ satisfy the following recurrence:

$$
H_{n+1}(x)=x H_{n}(x)-n H_{n-1}(x) .
$$

Hence combining (4.1) and (4.2) we see that

$$
w_{n}(x \mid r, 1)=\left(\frac{1+r}{1-r}\right)^{n / 2} H_{n}\left(\sqrt{\frac{1-r}{1+r}} x\right),
$$

for all $n \geq-1$. Thus we deduce that the one dimensional marginal has density equal to

$$
f_{X}(x \mid r, 1)=\sqrt{\frac{(1-r)}{2 \pi(1+r)}} \exp \left(-\frac{(1-r) x^{2}}{2(1+r)}\right) .
$$

3. Let $(Y, Z)$ be two random variables. Suppose that we know the marginal distribution of $Y$. The property that all conditional moments of order $n$ say of $Y$ are polynomials of the same order in conditioning random variable (i.e. $Z$ ) for $n$ $\geq 1$ was called general polynomial regression property (GRP) of $Y$ given $Z$ in [11]. Also, there the property that additionally the coefficients by the greatest powers (say $n$ ) are of the form $\rho^{n}$, where $\rho$ denotes here correlation coefficient between was called there simply polynomial regression (PR) property of $Y$ given $Z$. In [11] it was shown that if both marginals are standardized normal and both have GRP and one of then has PR property, then necessarily joint distribution of $(Y, Z)$ must be binormal distribution with parameters $(0,0 ; 1,1, \rho)$.

Theorem 2 states both, two random variables $(Y, Z)$ having joint distribution with the density $f_{Y Z}$ given by assertion ii) of Theorem 1 have GRP property. It seems that PR property is not satisfied by none of the variables.

However the intriguing question seems to be what other property of conditional moments should be additionally assumed in order to get the characterization of joint distribution $f_{Y Z}$ ?

Acknowledgement 1. The author is very grateful to two unknown referees. Especially to the one of them, who has carefully read and checked all formulae and pointed out typos and small mistakes. 


\section{REFERENCES}

[1] Ismail, Mourad E. H. Classical and quantum orthogonal polynomials in one variable. With two chapters by Walter Van Assche. With a foreword by Richard A. Askey. Encyclopedia of Mathematics and its Applications, 98. Cambridge University Press, Cambridge, 2005.

[2] Bryc, Włodzimierz. Stationary random fields with linear regressions. Ann. Probab. 29 (2001), no. 1, 504-519. MR1825162 (2002d:60014)

[3] Ismail, Mourad E. H.; Stanton, Dennis; Viennot, Gérard. The combinatorics of $q$-Hermite polynomials and the Askey-Wilson integral. European J. Combin. 8 (1987), no. 4, 379-392. MR0930175 (89h:33015) xviii+706 pp. ISBN: 978-0-521-78201-2; 0-521-78201-5 MR2191786 (2007f:33001)

[4] Szabłowski, Paweł J. Multidimensional $q$-normal and related distributions-Markov case. Electron. J. Probab. 15 (2010), no. 40, 1296-1318. MR2678392

[5] Szabłowski, Paweł J. Befriending Askey-Wilson polynomials, Infin. Dimens. Anal. Quantum Probab. Relat. Top. , Vol . 17, No. 3 (2014) 1450015 (25 pages), http://arxiv.org/abs/1111.0601

[6] Szabłowski, Paweł J. On the $q$-Hermite polynomials and their relationship with some other families of orthogonal polynomials. Demonstratio Math. 46 (2013), no. 4, 679-708. MR3136185, http://arxiv.org/abs/1101.2875.

[7] Szabłowski, Paweł J. On the structure and probabilistic interpretation of Askey-Wilson densities and polynomials with complex parameters. J. Funct. Anal. 261 (2011), no. 3, 635-659. MR2799574, http://arxiv.org/abs/1011.1541

[8] Szabłowski, Paweł J. q-Gaussian distributions: simplifications and simulations. J. Probab. Stat. 2009, Art. ID 752430, 18 pp. MR2602881

[9] Bryc, Włodzimierz; Matysiak, Wojciech; Szabłowski, Paweł J. Probabilistic aspects of AlSalam-Chihara polynomials. Proc. Amer. Math. Soc. 133 (2005), no. 4, 1127-1134 (electronic). MR2117214 (2005m:33033)

[10] Szabłowski, Paweł J. Around Poisson-Mehler summation formula. Hacet. J. Math. Stat. 45 (2016), no. 6, 1729-1742. MR3699734, http://arxiv.org/abs/1108.3024

[11] Bryc, Włodzimierz; Szabłowski, Paweł J. Some characteristic of normal distribution by conditional moments. Bull. Polish Acad. Sci. Math. 38 (1990), no. 1-12, 209-218. MR1194266

[12] Szabłowski, Paweł J., On generalized Kesten-McKay distributions, submitted, http://arxiv.org/abs/1507.03191

Emeritus in Department of Mathematics and Information Sciences, Warsaw UniverSity of Technology ul Koszykowa 75, 00-662 Warsaw, Poland 